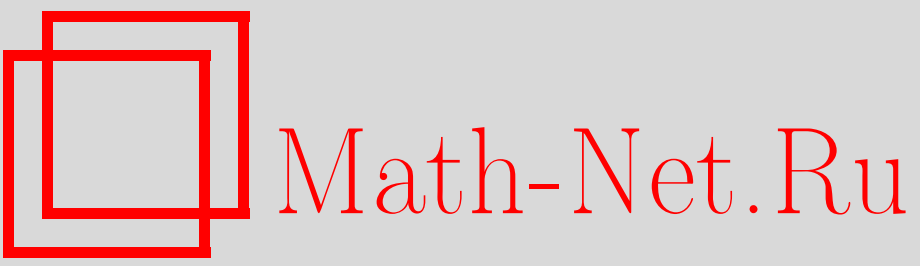

Л. К. Бакалинский, Динамика булева усреднения в одномерных решетчатых моделях с антиферромагнитным взаимодействием, ТМФ, 1998, том 117, номер 3, 435-441

DOI: https://doi.org/10.4213/tmf944

Использование Общероссийского математического портала Math-Net.Ru подразумевает, что вы прочитали и согласны с пользовательским соглашением

http://www. mathnet.ru/rus/agreement

Параметры загрузки:

IP: 52.23 .180 .231

26 апреля 2023 г., 12:45:46 


\title{
ДИНАМИКА БУЛЕВА УСРЕДНЕНИЯ В ОДНОМЕРНЫХ РЕШЕТЧАТЫХ МОДЕЛЯХ С АНТИФЕРРОМАГНИТНЫМ ВЗАИМОДЕЙСТВИЕМ
}

\begin{abstract}
Рассмотрена модель Хаббарда одномерного решетчатого газа с антиферромагнитным взаимодействием. Минимизация энергии этой модели осуществляется с помощью булева усреднения. Для случая произвольного начального распределения частиц газа на прямой показано, что зависимость концентрации газа от химического потенциала имеет вид канторовой лестницы.
\end{abstract}

\section{1. ВВЕДЕНИЕ}

Рассмотрим модель одномерного решетчатого газа [1]. Расположение частиц в нем описывается двусторонней последовательностью $\left(u_{n}\right): Z \rightarrow\{0,1\}$. Ячейки, для которых $u_{n}=1$, заняты частицами газа. Энергия $\gamma_{i}$ взаимодействия частиц, находящихся на расстоянии $i$ друг от друга, предполагается такой, что: $\gamma_{i}>0$ (антиферромагнитный характер взаимодействия); последовательность $\left(\gamma_{i}\right)$ монотонно убывает;

$$
\sum_{i=1}^{\infty} \gamma_{i}<\infty
$$

Энергия системы в модели Хаббарда $[2,3]$ может быть записана как формальный гамильтониан

$$
H=-\psi \sum_{i \in Z} u_{i}+\sum_{i \in Z, j \in Z, i>j} \gamma_{i-j} u_{i} u_{j},
$$

где $\psi$ - химический потенциал.

Вопросы минимизации энергии такой системы решались для периодических последовательностей в работах [1-4] разными способами. В [4] это делалось в духе динамического программирования при помощи булевой усредняющей процедуры (БУП), что позволяло снять требование выпуклости весовой последовательности $\left(\gamma_{i}\right)$, необходимое в подходе работ [1-3].

Следуя статье [4], рассмотрим задачу минимизации как задачу управления. Предположим, что все значения $u_{m}$ при $m<n$ определены; необходимо определить значение $u_{n}$

\footnotetext{
* Челябинский государственный педагогический университет, Челябинск, Россия
} 
так, чтобы энергия системы не увеличилась. Выделим в гамильтониане (1) только те слагаемые, которые содержат переменные $u_{n}$, и получим прирашение энергии

$$
\Delta H=u_{n}\left(\sum_{j<n} \gamma_{n-j} u_{j}-\psi\right) .
$$

Чтобы сделать $\Delta H$ неположительным, следует считать, что

$$
u_{n}=\left\{\begin{array}{cc}
1, \text { если } & \sum_{i=1}^{\infty} \gamma_{i} u_{n-i} \leqslant \psi ; \\
0, \text { если } & \sum_{i=1}^{\infty} \gamma_{i} u_{n-i}>\psi .
\end{array}\right.
$$

Соотношение (2) называется БУП. До сих пор были исследованы только периодические основные состояния в моделях одномерного решетчатого газа с антиферромагнитным взаимодействием [1-5]. В данной работе рассматриваются основные состояния, построенные с помощью БУП, при произвольном, не обязательно периодическом распределении частиц на прямой.

Числом врашения траектории $\left(u_{n}\right)$ будем называть долю единищ в обшем потоке значений $\left(u_{n}\right)$ при $n>0$. Для рассматриваемой модели одномерного решетчатого газа число вращения представляет собой концентрацию молекул газа. Необходимо показать, что число врашения последовательности $\left(u_{n}\right)$, порождаемой БУП, не зависит от значений $u_{n}$ при $n<0$, а зависит только от значения параметра $\psi$. Этот результат можно интерпретировать как доказательство того, что в динамике изменения решетчатого газа установившееся значение концентрации зависит только от химического потенциала.

\section{2. ПОСТАНОВКА ЗАДАЧИ И ОСНОВНОЙ РЕЗУЛЬТАТ}

Рассмотрим двустороннюю бинарную последовательность $\left(u_{n}\right): Z \rightarrow\{0,1\}$ такую, что для всех $n>0$ выполняется соотношение (2). Последовательность $\left(u_{n}\right)$ при $n \leqslant 0$ назовем начальным условием, а при $n>0$ - траекторией БУП. Число вращения $\omega$ траектории $\left(u_{n}\right)$ определим формулой

$$
\omega=\lim _{n \rightarrow \infty} \frac{1}{n} \sum_{i=1}^{n} u_{i} .
$$

Пусть $e_{1} e_{2} \ldots e_{l}$ - это слово в алфавите $\{0 ; 1\}$, его числом врашения назовем число

$$
\frac{1}{l} \sum_{i=1}^{l} e_{i}
$$

Введем понятие области сушествования цикла. Для этого рассмотрим начальное условие - периодическую последовательность с периодом $e_{1} e_{2} \ldots e_{l}$. Областью существования цикла $e_{1} e_{2} \ldots e_{l}$ назовем множество значений переменной $\psi$, при которых действие БУП (2) не нарушает периодичности начального условия (т.е. $u_{n}=u_{n+l}$ для всех $n \in Z)$. 
Для периодической траектории взвешенные суммы

$$
\sum_{i=1}^{\infty} \gamma_{i} u_{n-i}
$$

при различных $n$ принимают последовательно ровно $l$ различных значений:

$$
S_{1}=\sum_{j=1}^{l} \sum_{k=1}^{\infty} \gamma_{l k+1-j} e_{j}, \quad S_{i}=\sum_{k=1}^{i-1} \gamma_{k} e_{i-k}+\sum_{j=1}^{l} \sum_{k=1}^{\infty} \gamma_{l k+i-j} e_{j} \quad(2 \leqslant i \leqslant l) .
$$

Для того чтобы значение переменной $\psi$ принадлежало области сушествования цикла $e_{1} e_{2} \ldots e_{l}$, необходимо и достаточно выполнение следующих $l$ неравенств: $S_{i} \leqslant \psi$, если $e_{i}=1$, и $S_{i}>\psi$, если $e_{i}=0$. Поэтому непустая область сушествования цикла $e_{1} e_{2} \ldots e_{l}$ есть полуинтервал $\left[S^{\prime} ; S^{\prime \prime}\right)$, границы которого суть $S^{\prime}=\max \left\{S_{i}: e_{i}=1\right\} ; \quad S^{\prime \prime}=$ $\min \left\{S_{i}: e_{i}=0\right\}$.

В качестве циклов будем рассматривать слова, в которых единицы "равномерно распределены" среди нулей и наоборот. Таким словам разные авторы давали разные названия: штурмовы цепочки [6], равномерно 2-раскрашенные слова [7], хаббардовы конфигурации [1].Эти определения описывают один и тот же тип последовательностей [8]. Следуя работе [1], дадим определение хаббардовой конфигурации. Предварительно введем следующее определение: слово $A$, состоящее из нулей и единищ, является сдвигом слова $B$, если сушествуют такие слова $C$ и $D$, что $A=C D$ и $B=D C$.

ОПРЕДЕлЕНИЕ. Слово $e_{1} e_{2} \ldots e_{l}$ является хаббардовой конфигурацией, если в этом слове и в любом его сдвиге между любой единицей и $i$-й единицей справа от нее находится либо $[i / w]-1$, либо $[i / w]$ букв.

Для каждого рационального числа $w$ существует ровно одна (с точностью до сдвига) хаббардова конфигурация, число врашения которой равно $w$. Например, слова 01, 01011, 01011011 суть хаббардовы конфигурации с числами вращения $1 / 2,3 / 5,5 / 8$, соответственно. Однако слово 0011 с числом врашения $1 / 2$ не является хаббардовой конфигурацией.

Периодические траектории и начальные условия, периоды которых являются хаббардовыми конфигурациями, будем называть регулярными. Известно [5], что: 1) области сушествования хаббардовых конфигураций образуют на оси значений $\psi$ систему интервалов, дополнение к объединению этой системы интервалов есть множество нулевой лебеговой меры, гомеоморфное канторову; 2) если область сушествования цикла, не являющегося хаббардовой конфигурацией, непуста, то она лежит внутри области существования хаббардовой конфигурации с тем же числом врашения. Построенная таким образом зависимость числа вращения от химического потенциала $\psi$ имеет вид канторовой лестницы. Мы хотим показать, что при переходе к произвольным начальным условиям БУП зависимость числа врашения от химического потенциала не изменяется. Таким образом, мы докажем, что канторова лестница характеризует фазовые переходы в модели Хаббарда не только при периодических начальных условиях, как показано в [1-5], но и при произвольных начальных условиях. Этот факт непосредственно вытекает из следующей теоремы. 
ТЕОРемА 1. Если $\psi$ - внутренняя точка области существования иикла $e_{1} e_{2} \ldots e_{l}$, являющегося хаббардовой конфигурацией с числом вращения $w$, то для любой последовательности $\left(u_{n}\right)$, удовлетворяющей при $n>0$ условию (2), имеет место равенство

$$
w=\lim _{n \rightarrow \infty} \frac{1}{n} \sum_{i=1}^{n} u_{i} .
$$

Кроме того, мы покажем, что при любых значениях химического потенциала $\psi$ и при произвольном начальном распределении частищ на прямой число вращения траектории $\left(u_{n}\right)$ существует. Это следует из теоремы 2.

ТЕОрема 2. При всех значениях переменной $\psi$ для любой последовательности $\left(u_{n}\right)$, удовлетворяющей при $n>0$ условию (2), существует предел

$$
\omega=\lim _{n \rightarrow \infty} \frac{1}{n} \sum_{i=1}^{n} u_{i}
$$

\section{3. ДОКАЗАТЕЛЬСТВА ТЕОРЕМ}

Пусть в результате действия БУП образовалась траектория $\left(u_{n}\right), n \in N$. Если $\psi \leqslant 0$, то заключения теорем 1,2 очевидны. Рассмотрим случай $\psi>0$. Легко показать, что в этом случае в силу условия $\gamma_{i}>0$ множество единиц в последовательности $\left(u_{n}\right)$ при $n>0$ бесконечно. Построим функцию $f(n)$, аргументом которой является номер единицы в траектории, а значением - номер позиции, которую эта единица занимает в траектории. Например, если $\left(u_{n}\right)=0100101 \ldots$, то $f(1)=2, f(2)=5, f(3)=7$. В дальнейшем мы используем следуюшее свойство хаббардовой конфигурации, почти непосредственно вытекающее из ее определения: если $f(n)$ - функция, построенная по траектории $u_{n}^{\prime}$, соответствующей правой границе области сушествования хаббардовой конфигурации $e_{1} e_{2} \ldots e_{l}$ с числом врашения $w$, то график $f(n)$ всюду лежит вьше прямой $p^{\prime}(n)=n / w$, но прилегает к ней плотно (т.е. между прямой и графиком регулярной траектории нет узлов целочисленной решетки [5]).

ЛЕмма 1. Если $\psi<S^{\prime}$, где $S^{\prime}$ - правая граница области существования хаббардовой конфигурации $e_{1} e_{2} \ldots e_{l}$ с числом вращения $w$, то существует натуральное число $k$ такое, что для любого натурального п выполняется неравенство

$$
f(n) \geqslant \frac{n}{w}-\frac{k}{w}
$$

ДокАЗАТЕЛЬСтво. С геометрической точки зрения утверждение леммы означает, что сушествует прямая $p(n)=n / w-k / w$ такая, что график $f(n)$ всюду лежит выше этой прямой. Найдем число $r \in N$ такое, что

$$
\psi<\sum_{i=1}^{r} \gamma_{i} u_{m-i}^{\prime}
$$




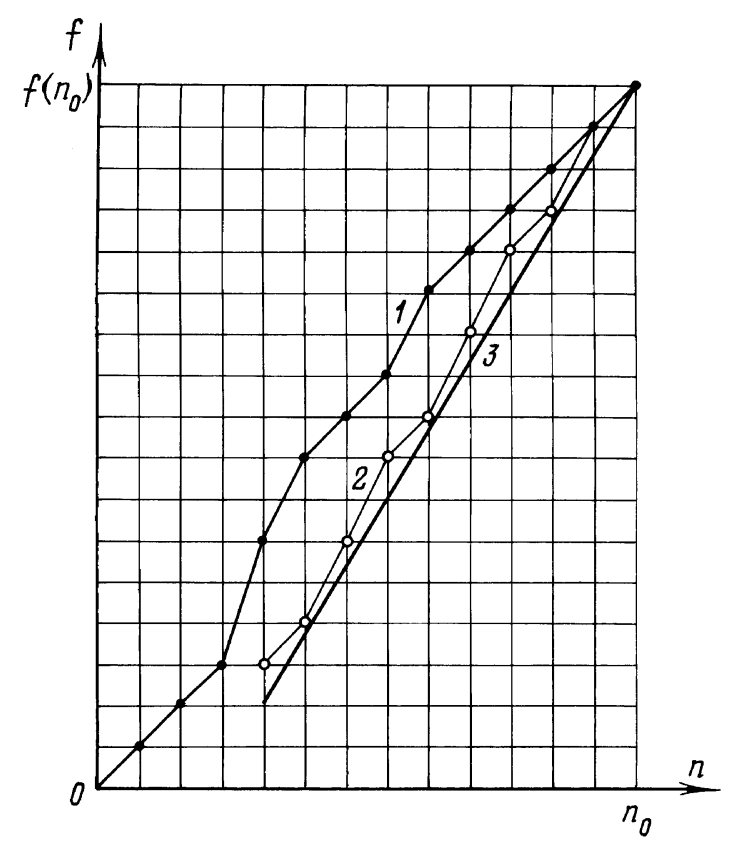

где $\left(u_{m}^{\prime}\right)$ - регулярная траектория, соответствуюшая $S^{\prime}$. Искомая константа $k$ равна

$$
\sum_{i=1}^{r} u_{m-i}^{\prime}
$$

Действительно, для всех значений $n \leqslant k$ неравенство (3), очевидно, выполняется. Предположим (для приведения к противоречию), что существуют значения $n>k$, для которых неравенство (3) не выполняется, и пусть $n_{0}-$ наименьшее из таких чисел. Тогда для всех натуральных $n$ из интервала $\left[n_{0}-k, n_{0}\right)$ вьполняется неравенство $f(n) \geqslant p(n)$ и тем более выполняется неравенство $f(n)>p_{1}(n)$, где $p_{1}(n)=\left(n-n_{0}\right) / w+f\left(n_{0}\right)$. Сравним сумму

$$
S=\sum_{i=1}^{\infty} \gamma_{i} u_{f\left(n_{0}\right)-i}
$$

с регулярной суммой

$$
S^{\prime}=\sum_{i=1}^{\infty} \gamma_{i} u_{m-i}^{\prime}
$$

Для этого (см. рисунок) отложим от точки $\left(n_{0} ; f\left(n_{0}\right)\right)$ назад график регулярной траектории $\left(u_{m}^{\prime}\right)$ (ломаная 2$)$, так чтобы сумма $S^{\prime}$ считывалась из этой точки аналогично сумме $S$. Этот график плотно прилегает к прямой $p_{1}(n)$ (линия 3 на рисунке). Поэтому график траектории $f(n)$ (ломаная 1 ) на отрезке длиной $k$ расположен не ниже графика регулярной траектории. Таким образом, если при считывании из точки $\left(n_{0} ; f\left(n_{0}\right)\right)$ выписать друг под другом ненулевые слагаемые сумм $S$ и $S^{\prime}$, то слагаемыми суммы $S$ 
будут члены последовательности $\left(\gamma_{i}\right)$ с номерами, не меньшими номеров соответствуюших слагаемых суммы $S^{\prime}$. В силу монотонного убывания весовой последовательности $\left(\gamma_{i}\right)$ получаем, что

$$
\psi<\sum_{i=1}^{r} \gamma_{i} u_{m-i}^{\prime} \leqslant \sum_{i=1}^{r} \gamma_{i} u_{f\left(n_{0}\right)-i}<\sum_{i=1}^{\infty} \gamma_{i} u_{f\left(n_{0}\right)-i}
$$

и, следовательно, $u_{f\left(n_{0}\right)}=0$, что противоречит построению функции $f(n)$. Лемма 1 доказана.

Рассмотрим теперь аналогично долю нулей в траектории. Подобно функции $f(n)$ построим функцию $g(n)$, ставящую в соответствие номеру нуля в траектории номер его позиции. Аналогично лемме 1 доказывается следующая лемма.

Лемма 2. Если $\psi>S^{\prime \prime}$, где $S^{\prime \prime}$ - левая граница области существования хаббардовой конфигурачии $e_{1} e_{2} \ldots e_{l}$ с числом вращения $w$, то существует такое натуральное число $k$, что для любого натурального $n$ выполняется неравенство

$$
g(n) \geqslant \frac{n}{1-w}-\frac{k}{1-w} .
$$

ДОКАЗАТЕЛЬСТВО ТЕОРЕМЫ 1. ИСходя из оПределений функций $f(n)$ и $g(n)$ число вращения может быть вычислено следующим образом:

$$
\omega=\lim _{n \rightarrow \infty} \frac{n}{f(n)}=1-\lim _{n \rightarrow \infty} \frac{n}{g(n)} .
$$

Из леммы 1 следует, что

$$
\lim _{n \rightarrow \infty} \sup \frac{n}{f(n)} \leqslant w
$$

а из леммы 2 -

$$
\lim _{n \rightarrow \infty} \sup \frac{n}{g(n)} \leqslant 1-w .
$$

Поэтому $\omega=w$. Теорема 1 доказана.

ДОКАЗАТЕЛЬСТВО ТЕОРЕМЫ 2. Если $\psi$ является внутренней точкой области существования цикла, то заключение теоремы 2 следует из теоремы 1 . В противном случае выберем возрастающую $\left(v_{j}^{\prime}\right)$ и убывающую $\left(v_{j}^{\prime \prime}\right)$ последовательности из внутренних точек областей существования циклов, сходящиеся к $\psi$. Точкам $v_{j}^{\prime}$ и $v_{j}^{\prime \prime}$ соответствуют числа врашения $w_{j}^{\prime}$ и $w_{j}^{\prime \prime}$. Тогда для любого $j$ выполняются неравенства

$$
\lim _{n \rightarrow \infty} \sup \frac{1}{n} \sum_{i=1}^{n} u_{i} \leqslant w_{j}^{\prime \prime}
$$

(лемма 1) и

$$
\lim _{n \rightarrow \infty} \inf \frac{1}{n} \sum_{i=1}^{n} u_{i} \geqslant w_{j}^{\prime}
$$

(лемма 2). Очевидно, что $\lim _{j \rightarrow \infty} w_{j}^{\prime}=\lim _{j \rightarrow \infty} w_{j}^{\prime \prime}$, откуда и следует наше утверждение. 


\section{4. ЗАКЛЮЧЕНИЕ}

Из доказательств теорем 1 и 2 следует, что предел устанавливается тем медленнее, чем сильнее выражено дальнодействие в модели. В случае же быстрого убывания весовой последовательности $\left(\gamma_{i}\right)$ (когда для любого $i$ выполняется неравенство $\gamma_{i+1} \leqslant \gamma_{i} / 2$ ) можно, кроме того, показать, что для почти всех значений химического потенциала и при любом начальном условии траектория БУП становится периодической.

\section{Список литературы}

[1] С. Е. Бурков, Я. Г. Синай. УМН. 1983. Т. 38. Вып. 4. С. 205-225.

[2] J. Hubbard. Phys. Rev. B. 1978. V. 17. P. 494-505.

[3] P. Bak, R. Bruinsma. Phys. Rev. Lett. 1982. V. 49. № 4. P. 249-251.

[4] М.М. Кипнис. Докл. РАН. 1994. Т. 336. Вып. 3. С. 316-319.

[5] M.M. Kipnis. Russ. J. Math. Phys. 1996. V. 4. № 3. P. 397-402.

[6] M. Morse, G.A. Hedlund. Amer. J. Math. 1940. V. 62. P. 1-42.

[7] V. Baladi, D. Rocmore, N. Tongring, C. Tresser. Nonlinearity. 1992. V. 5. P. 1111-1136.

[8] M. М. Кипнис. Теория булева усреднения для исследования явления равномерного 2-раскрашивания в системах управления, в преобразователях сигналов и в моделях статистической механики. Дисс. на соискание уч. ст. д-ра физ.-мат. наук. М.: Ин-т системного анализа РАН, 1995.

Поступила в редакцию 13. III.1998 г. после доработки 26.V.1998 г. 